\title{
RELAJACIÓN NEUROMUSCULAR EN CIRUGÍA LAPAROSCÓPICA. BUCARAMANGA-COLOMBIA
}

\author{
Márquez Benítez Ana ${ }^{1}$, Torres Wilches Héctor ${ }^{2}$, Meléndez Flórez Héctor ${ }^{3}$, \\ MD. Residente de Tercer Año - Posgrado de Anestesiología y Reanimación, Universidad Industrial de Sa. \\ Anestesiólogo, Magister en Epidemiología Clínica de la Universidad de la Frontera Temuco - Chile. \\ MD. Anestesiólogo - Intensivita, MSc Profesor Titular Escuela de Medicina, Universidad Industrial.
}

Introducción: La relajación neuromuscular en pacientes sometidos a cirugía laparoscópica es un pilar obligatorio en el manejo anestésico que permite garantizar un adecuado campo quirúrgico, menor presión intraabdominal y por consiguiente, menos cambios fisiológicos intraoperatorios que generen hipotensión, trastornos del ritmo, hipercapnia y aumento de la presión de la vía aérea ${ }^{1}$.

Objetivo General: Describir dentro del manejo de la Relajación Neuromuscular en Cirugía Laparoscópica: la incidencia de monitorización, grado de Bloqueo Neuromuscular (BNM) logrado, satisfacción del cirujano y comportamiento hemodinámico durante el neumoperitoneo.

Material y Métodos: Estudio descriptivo analítico de tipo corte transversal realizado en 108 pacientes que fueron llevados a cirugía laparoscópica electiva o de urgencia bajo anestesia general. Se recogieron pacientes desde diciembre de 2015 a septiembre de 2016 en 3 instituciones hospitalarias de la ciudad de Bucaramanga - Colombia. Se aplicó un instrumento de recolección para lo cual se midieron variables cualitativas y cuantitativas dentro de las cuales se describe la monitorización del BNM con aceleromiografía por parte del Anestesiólogo, grado de BNM, Satisfacción del Cirujano en 3 momentos del neumoperitoneo (inicio, 30 minutos y finalización) calificada como buena, regular, mala y complicaciones asociadas al neumoperitoneo durante el intraoperatorio como Hipotensión, Taquicardia / Bradicardia, Hipercapnia y Aumento de la Presión de la Vía Aérea. Se analizaron los datos con el paquete estadístico STATA.

Resultados: Se evaluaron 108 pacientes, edad media de 44,3 años [DE $=17,2$ años]). $75 \%$ de los pacientes fueron intervenidos por patología de la vía biliar. La incidencia de monitorización del BNM fue sólo del 21\%. En el momento de la creación del neumoperitoneo, el grado de BNM alcanzado fue Intenso $16,7 \%$, Profundo $13,9 \%$, Moderado 37,0\%, Recuperación < 90\% 27,8\% y Recuperación $90 \%$ 4,6\%. La Satisfacción del Cirujano con respecto a la Relajación Neuromuscular durante el neumoperitoneo fue documentada como Buena en el $88,9 \%, 90,7 \%$ y $92,6 \%$ correspondiente a los 3 momentos evaluados. Dentro de las complicaciones estudiadas se documentó Hipotensión Arterial 4,7\%, Bradicardia 40,7\%, Hipercapnia $11,1 \%$ y Aumento de la Presión de la Vía Aérea 40,7\%. Se identificó la asociación entre mayor grado de BNM y ocurrencia de hipercapnia $(\mathrm{p}<0,001)$ y bradicardia $(\mathrm{p}<0,036)$ al final del procedimiento.

Conclusiones: La monitorización del BNM en anestesia para cirugía laparoscópica sigue siendo una práctica subestimada, genera sesgos, aumenta riesgo de Bloqueo Neuromuscular Residual, y si se asocia a presión de vía aérea elevada intraoperatoria, ocasiona mayor morbilidad pulmonar posoperatoria. La Bradicardia y Presión de Vía Aérea elevada fueron las complicaciones más frecuentes en el estudio. Existe una asociación lineal estadísticamente significativa entre grado de BNM con bradicardia e hipercapnia, sin embargo, se necesitan más estudios para corroborarlo. Se evidenció una relación directa entre grado de BNM y satisfacción del cirujano.

\section{Referencias}

1. Madsen M, Staehr-Rye A, Gätke M. and Claudius C. Neuromuscular blockade for optimising surgical conditions during abdominal and gynaecological surgery: a systematic review. Acta Anaesthesiologica Scandinavica 2014.

No hubo financiación externa. 